\title{
IV. Envelopes
}

\section{The Neutral Shells of Planetary Nebulae}

\author{
Antonella Natta ${ }^{1}$ and David Hollenbach ${ }^{2}$ \\ ${ }^{1}$ Osservatorio di Arcetri, Firenze; ${ }^{2}$ NASA Ames Research Center, Moffett Field, California
}

Recent observations of a number of molecular lines, among them the $\mathrm{H}_{2} 2 \mu \mathrm{m}$ vibrational transitions, have clearly shown that in many planetary nebulae (PNe) a significant component of the eject mass is neutral.

We present in this paper an attempt to theoretically model the emission expected from neutral tori or shells surrounding the hot central stars of PNe. We include the effects of shocks, FUV photons, and soft $\mathrm{X}$ rays on the predominantly neutral gas, and hope to give insight into which of these three processes causes the $\mathrm{H}_{2} 2 \mu \mathrm{m}$ emission.

We assume that the ejected shell has constant mass and velocity and fills a fixed fraction of the solid angle seen from the star. The shell overtakes and shocks the red giant wind from the previous epoch of mass loss. After about $t_{0} \simeq 2000$ years, the shell has traveled $R_{0} \simeq 10^{17} \mathrm{~cm}$ and the central star has warmed to $T_{*} \sim 30,000 \mathrm{~K}$, initiating a rapid rise in the luminosity of FUV photons, and of Lyman continuum photons. A three-layered shell is produced with an inner HII region, a central HI region, and an outer $\mathrm{H}_{2}$ region. The evolutionary tracks of Schönberner $(1983$, ApJ 272, 708) are adopted to follow the time dependence of the stellar radiation field. We follow the time dependent chemistry for $\mathrm{H}_{2}$, solving for the chemical and temperature structure and the emergent spectrum of the evolving shell (Hollenbach \& Natta 1995, ApJ 455, 133).

The results of our calculations show that we can identify three phases in the evolution of a typical PN.

i) The early evolution (at about the peak of $\Phi_{U V}$ ) is characterized by strong emission (more than $10^{-3} \mathrm{erg} \mathrm{cm}^{-2} \mathrm{~s}^{-1} \mathrm{sr}^{-1}$ ) in the $H_{2} 1-0 \mathrm{~S}$ (1) line due to thermal emission in the dense, warm molecular' gas. The shell is mostly molecular. ii) The next phase is characterized by fluorescent $\mathrm{H}_{2}$ emission by a relatively cool neutral gas. In this phase, the PN has a typical surface brightness in the 1-0 S(1) line of $\sim 10^{-4} \mathrm{erg} \mathrm{cm}^{-2} \mathrm{~s}^{-1} \mathrm{sr}^{-1}$. The ratio 2-1 S(1)/1-0 S(1) approaches the typical value for fluorescent emission of 0.5 . iii) At even later times, the $\mathrm{H}_{2}$ emission is dominated by thermal emission from the hot, X-ray heated gas. The ratio 2-1 S(1)/1-0 S(1) is in the range $\sim 0.2$ to $\sim 0.5$.

The duration of these phases depends on the evolution time scale of the central star, and, to a lower degree, on the properties of the shell. 